\title{
Addressing Quality of Life Issues in Long Term Survivors of Head \& Neck Cancer treated with Radiation Therapy
}

Bishan Basu ${ }^{1}$

\section{ABSTRACT}

The rapid advancement of curative treatment modalities has resulted in improvement of cure rates of head neck cancer leaving us with a larger number of long term survivors from the disease. Unfortunately, long term complications of therapy continue to hurt patients even after cure, compromising their quality of life. This is particularly true for the patients treated with primary radiation/chemo-radiation therapy, where so called organ preservation does not necessarily translate into preservation of organ function. Long term sequelae of treatment, particularly xerostomia and swallowing difficulties compromise the survivors' quality of life. More studies, particularly suited to our clinical scenario, are warranted to address the quality of life issues in these patients, so that better evidence-based guidelines may be developed for their benefit.

Keywords:

Head and Neck Neoplasms; Quality of Life; Xerostomia

$\mathrm{T}$ The effectiveness of standard cancer treatments targeted at improving cure or extending survival rates in patients suffering from head and neck cancer is tempered by long-term patient morbidity manifested as side effects. These long-term sequelae of treatment interfere a great deal with both physical and psychological functions compromising the quality of life of the long-term survivors.

The World Health Organization (WHO) defines Quality of Life (QoL) as "individuals' perception of their position in life in the context of the culture and value systems in which they live and in relation to their goals, expectations, standards and concerns. It is a broad ranging concept affected in a complex way by the person's physical health, psychological state,

1 - Department of Radiotherapy, Bankura Sammilani

Medical College, Bankura, West Bengal

\section{Corresponding author:}

Dr Bishan Basu

email: bishanbasu@gmail.com level of independence, social relationships, personal beliefs and their relationship to salient features of their environment." ${ }^{\prime \prime}$ But, how can we apply this allencompassing definition to address the quality of life issues in a long term survivor of head and neck cancer who has been treated with radiation therapy?

To elaborate it further, what are the most likely concerns of a patient who has suffered from head and neck malignancy, concerns which compromise his quality of life - a patient, whose disease is presently under control, who is under regular follow-up and who, though his disease is not hurting him anymore, is still suffering from the adverse reactions of his anti-cancer treatment?

Worldwide, there are approximately 560,000 new cases of head and neck cancer diagnosed and 300,000 deaths each year ${ }^{2}$ and among them, as many as $57.5 \%$ are in Asia, especially in India. ${ }^{3}$ Each year, over 200,000 new cases of head and neck malignancies are detected in India and here, it is the commonest malignancy. ${ }^{4}$ Compared to 
malignancies of other sites like lung, patients suffering from cancers of the head and neck region tend to fare better. In one population-based study, 5-year survival for localised head and neck cancers ranged from $52.9 \%$ to $80.2 \%$ depending on the subsite - cancers of the lip, mouth, nasopharynx and larynx had a better prognosis; however, less than one-fourth of cancers were localised in the organ of origin at diagnosis. Those with regional spread of disease experienced a threefold increased risk of death, and those with distant metastasis experienced a six-fold excess risk. ${ }^{5}$

So, in India, we get a lot of new patients suffering from head and neck malignancies; and a fair portion of these new patients' disease would be controlled or cured to get us a large number of long-term survivors. As newer modalities of treatment come into play, probability of cure / long term control would increase further.

Treatment of head and neck malignancies include surgery, radiotherapy and chemotherapy; very often all three of them are used sequentially or in combination. Treatment is becoming increasingly complex, prolonged and toxic. Each improvement in treatment modality has resulted in improvement of survival rates. For example, addition of chemotherapy (concurrent chemoradiation) has resulted in $6 \%$ to $8 \%$ improvement in five-year survival and 3\% improvement with altered fractionation (i.e., hyperfractionation or accelerated fractionation) schedules over the conventional radiation therapy protocol. ${ }^{6}$

In general, patients who have received radiation therapy tend to have a poorer quality of life score compared to the patients who have not. ${ }^{7}$ The modalities mentioned above - i.e., altered fractionation and concurrent chemoradiation - to improve the results of radiation therapy - are substantially more toxic compared to the conventional therapy; therefore, these modalities can potentially deteriorate the long term survivors' QoL further. Though studies comparing long-term residual and late adverse effects are not very common, one study on acute toxicities showed that the relative risk for acute toxicity of concurrent chemoradiotherapy, as compared to conventional radiation therapy, may be 320 and accelerated radiation therapy (concomitant boost technique), if combined with concurrent chemotherapy, may result in a relative risk of 590. The study stratified risks as low (100-140), moderate (150-390), high (400$490)$ and extreme $(>500){ }^{8}$

Studies show that almost all patients of head and neck malignancies have some general emotional and functional impairment due to their treatment; however, there are some treatment-specific problems which affect their quality of life. While surgically treated patients were more likely to suffer from long-standing pain and concerns about disfigurement, patients treated with radiation therapy (with or without concomitant chemotherapy) complain of dry mouth (xerostomia) and dysphagia. ${ }^{9,10}$ Here, we are going to discuss two most common post-radiotherapy complications which usually affect the long-term survivors' quality of life.

\section{Xerostomia}

Xerostomia or dry mouth is a complex problem having both an objective as well as a subjective component. After radiation therapy, salivary flow decreases. But the relationship between this decreased salivary flow and patient-reported dry mouth is not always a linear one. While xerostomia is often defined as reduction of salivary flow to less than or equal to $25 \%$ of baseline, a better definition should address the subjective component as well. Oxford Textbook of Palliative Medicine defines xerostomia as "the subjective feeling of dryness of the mouth, not always accompanied by a detectable decrease in saliva flow." 11

Patients treated with radiotherapy for head and neck cancer often suffer from xerostomia from the beginning of treatment - very often, this dry mouth continues and increases through the course of treatment and persists as permanent xerostomia. Radiation can affect one or both parotid glands and the submandibular salivary glands, resulting in a marked diminution in the normal salivary flow as a consequence of inflammation and degeneration of the acini and ducts, connective tissue and vascular components of the salivary glands. The 
most important factor affecting salivary flow after a curative dose of radiotherapy is the volume of the major salivary glands irradiated, particularly the parotid as it is more radiosensitive than the other major salivary glands.

In addition to gross reduction of salivary outflow, irradiation of the salivary glands causes saliva to become more viscous and acidic, with a loss of organic and inorganic components. Production of the aqueous component of whole saliva is much more sharply depressed than that of the protein component, leading to a more predominant subjective dryness. ${ }^{12}$

Xerostomia may give rise to a myriad of symptoms as saliva is vital for normal oral function. Symptoms due to hyposalivation can profoundly decrease quality of life. Saliva plays an important role in moistening food to allow bolus formation. Even mild xerostomia can result in a significant decrease in the variety and types of food that patients can eat. Difficulty forming a food bolus makes deglutition difficult. Patients, therefore, avoid eating and this may compromise their nutrition. Saliva maintains oral flora, thus preventing the development of dental caries. It lubricates mucosal membranes allowing normal speech and swallowing. Finally, xerostomia results in mucosal irritation and pain.

Apart from flow rate measurements, the level of amylase seems to be the best indicator of salivary gland function during radiotherapy, whereas albumin and lactoferrin are good indicators of the inflammatory reactions often related to irradiation. ${ }^{12}$ However, a self reported xerostomia-specific questionnaire may be the best tool to assess long term xerostomia in patients receiving radiation therapy for head and neck cancer. Two most popular patient-reported tools have been a six-item linear analog scale ${ }^{13}$ and the eight-item University of Michigan XQ. ${ }^{14}$ Additionally, all head and neck cancer related questionnaires have at least one item related to xerostomia.

As there is no proven efficacious treatment of xerostomia, prevention is the only option. Sparing at least a part of one parotid gland from exposure to radiation should be the goal, whenever feasible.
This is possible, particularly but not exclusively, with Intensity Modulated Radiation Therapy (IMRT). Often, when treatment of unilateral neck node is sufficient, opposite side parotid gland may be spared by simpler conformal techniques. However, one should not be overenthusiastic, as at least one study linked local recurrence to parotid sparing, demonstrating the importance of careful patient selection. ${ }^{15}$ Radioprotectants like amifostine have also been used and found to be useful.

A randomised controlled trial tested the efficacy of amifostine in patients with head and neck cancer. The subjects received standard fractionated radiation with or without amifostine (Ethyol), administered at $200 \mathrm{mg}$ per square metres as a 3 -minute IV infusion 15 to 30 minutes before each fraction of radiation. ${ }^{16}$ Patient eligibility criteria included that the radiation field encompassed at least $75 \%$ of both parotid glands. The incidence of acute xerostomia (90 days from the start of radiotherapy) and late xerostomia ( 9 to 12 months after radiotherapy) was significantly reduced in patients receiving amifostine. Whole saliva collection 1 year following radiation therapy showed better saliva production in the amifostine group. ${ }^{16}$ At 2 year follow-up, patients treated with amifostine had lesser subjective feeling of dry mouth and had more meaningful unstimulated salivary flow. ${ }^{17}$ But, the inconvenience of daily administration of the drug minutes before radiotherapy and the risk of potentially life-threatening adverse reactions like severe hypotension have limited the popularity of the drug.

Another way to prevent xerostomia was to surgically transplant one submandibular gland outside the radiation portal area. During the primary surgery, the contralateral submandibular gland was replanted in the submental region in selected patients, in whom submental region was supposed to be spared or shielded during post-operative radiation therapy. This approach prevented radiation-induced xerostomia in as many as $83 \%$ of study population after two years of radiation therapy. ${ }^{18,19,20}$ However, in the largest study, it was seen that around thirty per cent of the study population underwent the procedure unnecessarily - i.e. either radiation therapy was not given or if given, submental 
space could not be shielded. ${ }^{21}$

Once xerostomia develops, it is very difficult to treat. Muscarinic agonist sialogogues like pilocarpine has been used and was found to be beneficial. Pilocarpine, in a dose of $5 \mathrm{mg}$ thrice daily, provides best benefits with acceptable adverse effects. Best results are produced when the drug was used for more than 8 weeks. ${ }^{22-26} \mathrm{~A}$ recent study showed that the benefit of pilocarpine was independent of the dose and technique of radiation therapy as well as the volume of salivary gland under radiotherapy. ${ }^{27}$ This suggests that, in addition to residual undamaged major salivary gland parenchyma, pilocarpine might exert its main effect through its action on the minor salivary glands. Other adjunctives such as artificial saliva containing carboxymethylcellulose as a base are not helpful. Agents, which supposedly act directly on the salivary glands like anethole-trithione are not found be beneficial either. Proper counselling, psychological support and dietary advice to take of foods with high moisture content and the drinking of plenty of liquids with meals to facilitate mastication would work better.

\section{Swallowing Difficulties (Dysphagia \& Odynophagia)}

A major proportion of patients treated with radiation therapy, particularly with concomitant chemoradiation therapy would suffer from long-term problems with their swallowing abilities.

Swallowing is a complex series of mechanical processes that can be broken down into several phases: oral, pharyngeal, and oesophageal. The oral phase can be described as having two separate components - the oral preparation phase and the oral transport phase. During the oral phase, the lips and tongue play a vital role in oral bolus preparation and bolus propulsion to the oropharynx. During the subsequent pharyngeal phase, the tongue acts as the driving force for the food bolus while a complex sequence of physiologic processes propels the bolus towards the oesophagus. The oesophageal phase begins at the cricopharyngeal juncture or upper oesophageal sphincter and ends at the gastroesophageal juncture or lower oesophageal sphincter. Oesophageal peristalsis carries the bolus to the stomach. Deficits in any one of these phases can result in significant levels of disability.

Various instrumental techniques have been used to study swallowing - including electromyography, manometry, scintigraphy, ultrasound, endoscopy, and videofluoroscopy. However, the gold standard for swallowing assessment is videofluoroscopy, also referred to as the modified barium swallow study.

But, to assess the swallowing difficulties in any longterm survivor of head and neck cancer, particularly those treated with radiation therapy, it should be remembered that the inefficient swallowing reported by the patient may be a perceptive difficulty. Particularly, xerostomia diminishes a patient's perception of swallowing abilities, regardless of preserved swallowing physiology. ${ }^{28}$ On the other hand, the most severe complication of swallowing difficulties, i.e., aspiration is under-recognised as well as under-reported among survivors of head and neck cancer since the aspiration is usually silent in nature. ${ }^{29}$

Among patients who suffer from a 'true' disorder of swallowing, persistent oedema following radiation therapy and the development of fibrosis result in mechanical alterations in the deglutition process. Swallowing abnormalities can be seen in the oral preparation, oral, pharyngeal, and oesophageal phases. The most commonly identified swallowing abnormalities include decreased tongue base retraction, decreased laryngeal elevation, decreased epiglottic inversion, decreased pharyngeal wall motion and aspiration. Fortunately, in many cases, patients are able to tolerate oral diets with various modifications to prevent aspiration and improve the efficiency of swallowing. ${ }^{30}$

The prevalence of swallowing difficulties among long-term survivors of head and neck cancer is difficult to assess, as not many studies have yet addressed this issue. One study concluded that all patients had some degree of swallowing problems after 12 months of completion of radiation therapy, though this study did not assess the prevalence of swallowing disorder in the 
study population before the initiation of therapy. ${ }^{31}$ Once xerostomia is ruled out as the potential reason behind the swallowing difficulties, rehabilitative swallowing therapy works well.

It is very important that swallowing difficulties are recognized and treated early, as xerostomia in addition to swallowing difficulties, compromise nutrition of the patient. This is often aggravated by the fact that exposure to radiation can cause alteration of taste sensation, which may lead to aversion to food further compromising nutrition. Swallowing difficulties are best described by the patients and therefore, should best be assessed by patient-reported tools e.g. patient-reported outcome questionnaire. Unfortunately, commonly used head and neck quality of life measurement tools do not address this issue properly. Perhaps, we are in need for better assessment tools. M.D. Anderson Dysphagia Inventory and SWAL-QoL/SWAL-CARE are some of the experimental tools developed specifically for this purpose..$^{32-35}$ In addition to the patient's perspective on his swallowing difficulties, SWAL-QoL went one step further to include the care-givers' perspective. However, adaptation of these tools for the Indian socioeconomic and clinical scenario can be a challenge.

\section{Quality of Life Issues}

With our conventional clinical approach, it is most satisfying for us when we are able to cure a patient. But, not infrequently, the long term sequelae of cancer therapy can be really debilitating - often hurting more than the initial symptoms of the disease. Unfortunately, we are not always eager to take care of these long term problems. It is very uncommon to find a clinician who addresses the long term sequelae-related quality of life issues with the same eagerness as shown for curative therapy. But, we should better keep in mind that, whether from the disease itself or from the adverse effects of treatment, even after cure, it is the same patient who is still suffering. And it is hardly enough consolation for the patient to realize that though he is still suffering, his disease is cured.
At the beginning of therapy, the fear of cancer may so overwhelm the patient that the prospect of cure is really lucrative. Once acute toxicities subside, the patient may feel better and quality of life may improve. This is manifested in the studies where it is seen that patients' quality of life improves gradually after completion of therapy. Studies report that though quality of life is impaired on the initial days of therapy (e.g. at three months), it gradually improves afterwards (e.g. at one year). ${ }^{36,37}$

Though long term quality of life studies on survivors of head and neck cancer is not common, one such study has suggested a late decline in survivors' QOL scores at 10 years after treatment as compared with 1- and 2-year follow-up. ${ }^{38}$ In studies where decline in QoL score were not seen, stabilization of QoL at one year was commonly reported with negligible improvement in treatment-related complications over the years. ${ }^{39-48}$

In judging the efficacy of treatment, extending survival does not always correlate with improvements in QoL. This is particularly true in context of curative treatment for head and neck cancer. Conversely, specific treatments may not necessarily prolong life, but may enhance its quality. In developed world, public and private organizations have brought attention to the need for research addressing the issues of cancer survivors. Efforts have been made for preparing proper guidelines to serve the long term cancer survivors ${ }^{49}$, so that management of the long term complications may be evidence-driven. In fact, the Institute of Medicine, USA has stated clearly in their guidelines that : "Health care providers should use systematically developed evidence-based clinical practice guidelines, assessment tools, and screening instruments to help identify and manage late effects of cancer and its treatment. Existing guidelines should be refined and new evidence-based guidelines should be developed." ${ }^{50}$

In a developing country like ours - where patients suffering from head and neck cancer belong to a lower 
socioeconomic status and long term complications almost always mean loss of job - need of such guidelines is still more.

Unfortunately, in our country, major attention is driven towards the curative treatment and supportive care is invariably neglected, though supportive treatment usually requires less funding and may be more cost-effective. But, before development of such guidelines specifically suited to our clinical scenario, more studies addressing the quality of life issues in long term survivors in our country are necessary.

However, even in teaching institutions, compared to studies on curative treatment, very little research work is done to address the quality of life issues. With the recent advancement of curative treatment modalities, the improved possibility of cure is leaving an increasing number of long term survivors left with long term sequelae of therapy. We are looking forward to more research work addressing the quality of life issues among these long term survivors. Particularly, the young generation of clinicians entering into their training on the treatment of head and neck cancer should take more interest into the matter.

The role of Patient Reported Outcome (PRO) questionnaires as tools of quality of life measurement can never be overemphasized. A number of such PROs have been developed. Overviews of such PROs and guidelines on their use for the purpose of clinical trials have also been published. ${ }^{51-54}$ Translations as well as cross-cultural adaptations of such PROs and their validation studies can be a focus of research works in the coming years in the tertiary care teaching hospitals in our country. PROs should be used as tools to measure QoL only after such validation. Meanwhile, broad Qol tools like EORTC QLQ-C30 and HN35 may be used for the purpose of studies addressing quality of life issues.

\section{References}

1. Available at: http://www.who.int/mental_health/media/68.pdf ; accessed 21st December, 2014

2. Boyle P et al. World Cancer Report 2008. International Agency for Research on Cancer.

3. Chaturvedi P. Head and Neck Surgery; J Can Res Ther 2009: 5: 143

4. Kulkarni M R : Head and Neck Cancer Burden in India; Int J Head Neck Sur; Jan-Apr (2013); 4(1); 29-35

5. Yeole BB, Sankaranarayanan R, Sunny M Sc L, Swaminathan R, Parkin DM.; Survival from head and neck cancer in Mumbai (Bombay), India. Cancer. 2000 Jul 15;89(2):437-44.

6. Pignon JP, le Maitre A, Bourhis J; Meta-analysis of chemotherapy in head and neck cancer (MACH-NC): an update: Int J Radiat Oncol Biol Phy 2007;69(2 Suppl):S112-14

7. Martino R; Evaluation of Quality of Life and organ function in head and neck squamous cell carcinoma; Hematol Oncol Clin N Am; 22 (2008); 1239-1256

8. Trotti A, Pajak TF, Gwede CK et al; TAME : development of a new method for summarizing adverse effects of cancer treatment by the Radiation Therapy Oncology Group.; Lancet Oncol 2007; 8(7); 613-24

9. Chandu A, Smith AC, Rogers SN; Health-related Quality of Life in Oral Cancer : a review; J Oral Maxillofac Sur 2006; 64(3); 495-502

10. Nguyen NP, Sallah S,Karlsson U. et al; Combined chemotherapy and radiation therapy for head and neck malignancies: quality of life issues; Cancer 2002; 94(4); 1131-1141

11. De Conno F, Sbanotto A, Ripamonti C et. al.; Chapter 8.12 Mouth Care in Oxford Textbook of Palliative Medicine, 3rd Edition; Ed : Doyle D, Hanks G, Cherny NI, Calman K; Oxford University Press 2004

12. Makkonen, T.A. et al. Changes in the protein composition of whole saliva during radiotherapy in patients with oral or pharyngeal cancer. Oral Surgery 1986 62, 270-5.

13. Johnson JT, Ferretti GA, Nethery WJ et. al.; Oral Pilocarpine for post-irradiation xerostomia in patients with head and neck cancer. N Engl J Med 1993; 329(6): 390-5

14. Eisbruch A, Kim HM, Terrell JE et al. Xerostomia and its predictors following parotid-sparing irradiation of head-andneck cancer. Int J of Radiat Oncol Biol Phys 2001 50(3); 695704.

15. Cannon DM, Lee NY; Recurrence in region of spared parotid gland after definitive intensity-modulated radiotherapy for head and neck cancer: Int J Radiat Biol Oncol Phys 2008; 70(3): $660-5$

16. Brizel DM, Wasserman TH, Henke $M$ et al. Phase III randomized trial of amifostine as a radioprotectant in head and neck cancer; J Clin Oncol 2000; 18 (19): 3339-45

17. Wasserman TH, Brizel DM, Henke $M$ et.al.; Influence of intravenous amifostine on xerostomia, tumor control, and survival after radiotherapy for head-and-neck cancer: 2 year 
follow-up of a prospective, randomized, phase III trial. Int J Radiat Biol Oncol Phys 2005; 63(4): 985-90

18. Jha N, Sekaly H, McGaw T et. al.; Submandibular salivary gland transfer prevents radiation-induced xerostomia; Int $\mathrm{J}$ Radiat Oncol Biol Phys 2000; 46(1); 7-11

19. Seikaly H, Jha N, Harris JR et. al.: Long-term outcomes of submandibular gland transfer for prevention of post-radiation xerostomia; Arch Otolaryngol Head Neck Surg 2004; 130(8); 956-61

20. Seikaly, H. et al.. Submandibular gland transfer: a new method of preventing radiation-induced xerostomia. Laryngoscope $2001 ; 111$ (2), 347-52

21. Jha N, Seikaly H, Harris J et. al.; Prevention of radiation induced xerostomia by surgical transfer of submandibular salivary gland into the submental space; Radiother Oncol 2003; 66(3); 283-9

22. Schuller, D.E. et al.; Treatment of radiation side-effects with oral pilocarpine. J Surg Oncol 1989; 42; 272-6.

23. Greenspan D, Daniels T.E.; Effectiveness of pilocarpine in postradiation xerostomia. Cancer 1987; 59, 1123-5.

24. LeVeque, F.G. et al.. A multicenter, randomized, double-blind, placebo-controlled, dose-titration study of oral pilocarpine for treatment of radiation-induced xerostomia in head and neck cancer patients. J Clin Oncol 1993; 11; 1124-31.

25. Johnson, J.T. et al. Oral pilocarpine for post-irradiation xerostomia in patients with head and neck cancer. N Engl J Med $1993 ; 329 ; 390-5$.

26. Rieke, J.W. et al. Oral pilocarpine for radiation-induced xerostomia: integrated efficacy and safety results from two prospective randomized clinical trials. Int J Radiat Oncol Biol Phys 1995; 31; 661-9.

27. Horiot, J.C. et al.; Post-radiation severe xerostomia relieved by pilocarpine: a prospective French cooperative study. Radiother Oncol 2000; 55 (3), 233-9.

28. Logemann JA, Pauloski BR, Rademaker AW, et al. Xerostomia: 12-month changes in saliva production and its relationship to perception and performance of swallow function, oral intake, and diet after chemoradiation. Head Neck 2003; 25(6):432-7.

29. Nguyen NP, Frank C, Moltz CC, et al. Aspiration rate following chemoradiation for head and neck cancer: an underreported occurrence. Radiother Oncol 2006;80(3):302-6.

30. Dowling E.R., Murphy B., Cmelak, A.. Swallowing deficits in patients treated with combined chemoradiation for locally advanced head and neck cancer; Proceedings of the American Society of Clinical Oncology 2001; 20;227a.

31. Kendall K.A.; Structural mobility in deglutition after single modality treatment of head and neck carcinomas with radiotherapy. Head \& Neck 1998; 20; 720-5.
32. Chen AY, Frankowski R, Bishop-Leone J, et al. The development and validation of a dysphagia-specific quality-oflife questionnaire for patients with head and neck cancer: the M.D. Anderson dysphagia inventory. Arch Otolaryngol Head Neck Surg 2001;127(7):870-6.

33. McHorney CA, Bricker DE, Kramer AE, et al. The SWALQOL outcomes tool for oropharyngeal dysphagia in adults: I. Conceptual foundation and item development. Dysphagia 2000;15(3):115-21.

34. McHorney CA, Bricker DE, Robbins J, et al. The SWAL-QOL outcomes tool for oropharyngeal dysphagia in adults: II. Item reduction and preliminary scaling. Dysphagia 2000;15(3):12233.

35. McHorney CA, Robbins J, Lomax K, et al. The SWAL-QOL and SWAL-CARE outcomes tool for oropharyngeal dysphagia in adults: III. Documentation of reliability and validity. Dysphagia 2002;17(2):97-114.

36. Curran D, Giralt J, Harari PM, et al. Quality of life in head and neck cancer patients after treatment with high-dose radiotherapy alone or in combination with cetuximab. J Clin Oncol 2007;25(16):2191-7.

37. Ringash J, Lockwood G, O'Sullivan B, et al. Hyperfractionated, accelerated radiotherapy for locally advanced head and neck cancer: quality of life in a prospective phase I/II trial. Radiother Oncol 2008;87(2):181-7.

38. Mehanna HM, Morton RP. Deterioration in quality-of-life of late (10-year) survivors of head and neck cancer. Clin Otolaryngol 2006;31(3):204-11.

39. Bjordal K, Ahlner-Elmqvist M, Hammerlid E, et al. A prospective study of quality of life in head and neck cancer patients, part II: longitudinal data. Laryngoscope. 2001;111(8):1440-1452

40. Hammerlid E, Ahlner-Elmqvist M, Bjordal K, et al. A prospective multicentre study in Sweden and Norway of mental distress and psychiatric morbidity in head and neck cancer patients. Br J Cancer. 1999;80(5-6):766-774

41. Hammerlid E, Silander E, Hörnestam L, Sullivan M. Healthrelated quality of life three years after diagnosis of head and neck cancer-a longitudinal study. Head Neck. 2001;23(2):113-125

42. Hammerlid E, Taft C. Health-related quality of life in long-term head and neck cancer survivors: a comparison with general population norms. Br J Cancer. 2001;84(2):149-156

43. Abendstein $H$, Nordgren $M$, Boysen $M$, et al. Quality of life and head and neck cancer: a 5 year prospective study. Laryngoscope. 2005;115(12):2183-2192

44. Nordgren M, Abendstein H, Jannert M, et al. Health-related quality of life five years after diagnosis of laryngeal carcinoma. Int J Radiat Oncol Biol Phys. 2003;56(5):1333-1343 
45. Nordgren M, Jannert M, Boysen M, et al. Health-related quality of life in patients with pharyngeal carcinoma: a fiveyear follow-up. Head Neck. 2006;28(4):339-349

46. Bjordal K, Kaasa S, Mastekaasa A. Quality of life in patients treated for head and neck cancer: a follow-up study 7 to 11 years after radiotherapy. Int $\mathrm{J}$ Radiat Oncol Biol Phys. 1994;28(4):847-856

47. Bjordal K, Mastekaasa A, Kaasa S. Self-reported satisfaction with life and physical health in long-term cancer survivors and a matched control group. Eur J Cancer B Oral Oncol. 1995;31B(5):340-345

48. Bjordal K, Kaasa S. Psychological distress in head and neck cancer patients 7-11 years after curative treatment. Br J Cancer. 1995;71(3):592-597

49. Earle CC. Cancer survivorship research and guidelines: maybe the cart should be beside the horse. J Clin Oncol.

\section{7;25(25):3800-3801}

50. Institute of Medicine: From cancer patient to cancer survivor: Lost in transition. Washington, DC, National Academies Press, 2005

51. Rogers SN, Ahad SA, Murphy AP. A structured review and theme analysis of papers published on 'quality of life' in head and neck cancer: 2000-2005. Oral Oncol 2007;43(9):843-68.

52. Lipscomb J, Gotay CC, Snyder CF. Patient-reported outcomes in cancer: a review of recent research and policy initiatives. CA Cancer J Clin 2007; 57(5):278-300.

53. Lipscomb J, Reeve BB, Clauser SB, et al. Patient-reported outcomes assessment in cancer trials: taking stock, moving forward. J Clin Oncol 2007;25(32):5133-40.

54. Murphy BA, Ridner SH, Wells N, et al. Quality of life research in head and neck cancer: a review of the current state of the science. Crit Rev Oncol Hematol 2007;62(3):251-67. 